\title{
Dietas saudáveis e sustentáveis no âmbito do sistema alimentar no século XXI
}

\author{
Healthy and sustainable diets in the context of the food system in the \\ 21st century
}

Rozane Marcia Triches'

DOI: $10.1590 / 0103-1104202012622$

RESUMO O objetivo deste ensaio foi o de realizar análise reflexiva sobre o tema das dietas sustentáveis, salientando a necessidade de debater o que seria uma alimentação adequada, dado o impacto ambiental do sistema alimentar atual. Inicia-se com uma digressão sobre a construção dessa temática para, na sequência, traçar paralelos entre dietas saudáveis e sustentáveis. Para tanto, utilizaram-se bases de dados e literatura cinzenta. O conceito de dietas sustentáveis vem sendo construído com o objetivo de agregar às preocupações nutricionais, culturais e econômicas, as questões ambientais que envolvem todo o sistema alimentar, pensando a garantia de Segurança Alimentar e Nutricional no presente e futuro. No entanto, as pesquisas realizadas têm mostrado a complexidade em conjugar todas essas preocupações na formulação de uma dieta adequada. Em linhas gerais, autores estudados concordam que uma alimentação com baixo impacto ambiental é geralmente consistente com uma boa nutrição. Porém, mesmo dentro desses padrões alimentares, haverá alguns alimentos mais sustentáveis do que outros, baseados em fatores da cadeia de suprimentos, dependendo de como e onde os alimentos foram produzidos, fabricados e transportados. O campo científico da nutrição e as diretrizes e políticas alimentares devem dar a devida consideração à sustentabilidade ao estabelecer metas voltadas para a nutrição saudável.

PALAVRAS-CHAVE Dieta. Alimentação. Desenvolvimento sustentável. Saúde pública. Meio ambiente.

ABSTRACT The objective of this essay was to carry out a reflective analysis on the theme of sustainable diets, emphasizing the need to debate what would be an adequate diet, given the environmental impact of the current food system. It starts with a digression on the construction of this theme to, next, draw parallels between healthy and sustainable diets. For this purpose, databases and gray literature were used. The concept of sustainable diets has been built seeking to add nutritional, cultural and economic concerns to the environmental issues that surround the entire food system, thinking about the guarantee of Food and Nutrition Security in the present and future. However, pragmatically, research has shown the complexity in combining all these concerns in formulating an ideal diet. Overall, the study authors agree that a diet with low environmental impact is generally consistent with good nutrition. But even within these food patterns, there will be some foods that will be more sustainable than others, based on supply chain factors, depending on how and where food was produced, manufactured, and transported. The scientific field of nutrition and food guidelines and policies should give due consideration to sustainability when setting goals for healthy nutrition.

KEYWORDS Diet. Food. Sustainable development. Public health. Environment.

1 Universidade Federal da Fronteira Sul (UFFS) Realeza (PR), Brasil. rozane.triches@gmail.com 


\section{Introdução}

O tema das dietas sustentáveis vem sendo muito debatido nas últimas duas décadas, principalmente nos países europeus, ao passo que, no Brasil, sejam poucos os estudos existentes até o momento' ${ }^{1}$. Embora as questões relativas à soberania e segurança alimentar e nutricional, desenvolvimento sustentável, agroecologia, agricultura familiar, entre outros tópicos, estejam sendo abordadas mais enfaticamente na academia brasileira, o papel da dieta e do consumo alimentar na sustentabilidade e no direcionamento do sistema alimentar ainda permanece periférico ${ }^{1}$.

O desafio alimentar para o século XXI é bastante complexo e vai além de pensarmos apenas a saudabilidade das dietas. A alimentação vem tomando uma dimensão que afeta a nossa própria existência no planeta. As expectativas são que em 2050 já sejamos mais que 9 bilhões de habitantes e que o sistema alimentar se reproduzido nos moldes atuais não dará conta desse incremento, considerando a espoliação dos recursos naturais como água, solo e energia fóssil.

Nesse sentido, três paradoxos vêm sendo explorados. O primeiro, diz respeito à inabilidade que o sistema alimentar tem de oferecer alimentação adequada às pessoas. Há 868 milhões de pessoas que não têm acesso suficiente à comida e 1,5 bilhões que têm excesso de peso. O segundo pontua que quase $50 \%$ do que estamos produzindo está alimentando carros e animais. Um terço do que produzimos de alimentos vira ração, sendo que $40 \%$ da produção mundial de grãos é para essa finalidade ( $97 \%$ da soja e $40 \%$ dos cereais produzidos no mundo) e em torno de $7 \%$ torna-se biocombustível ${ }^{2}$. Finalmente, o terceiro ponto diz respeito à quantidade de alimentos desperdiçados que, segundo a Food and Agriculture Organization (FAO) ${ }^{3}$ somam em torno de um terço da produção, o que poderia estar alimentando quatro vezes o número de pessoas que passam fome no mundo.

Outro ponto importante é que a pegada ecológica, que mede o uso de superfície de terra e água biologicamente produtivas, necessárias para produzir recursos e absorver resíduos ${ }^{4}$, atual é referente a um planeta e meio. Portanto, usa-se mais do que a possibilidade de regeneração dos recursos naturais, e a tendência para 2050 é de alcançar três planetas. Rockström et al. ${ }^{5}$ já indicavam em 2009 que já havíamos ultrapassado três limites planetários, dentro dos quais espera-se que a humanidade possa operar com segurança: o limite das mudanças climáticas, o da taxa de perda de biodiversidade e o das mudanças no ciclo global do nitrogênio.

Tal constatação se liga diretamente com o sistema alimentar, visto que a produção de alimentos atual é responsável por 20 a 30\% da emissão de Gases do Efeito Estufa (GEE); cerca de $24 \%$ da área fértil apresenta uma ou outra forma de degradação do solo em função do modelo agrícola adotado; a utilização de terras para cultivos de monoculturas e de pecuária tem gerado o desflorestamento e perda de grande parte da biodiversidade; e a água está sendo contaminada, incluindo a água do mar, levando a desequilíbrios no ambiente marinho. No entanto, não só a produção de alimentos gera problemas ambientais, mas também os processos envolvidos com a cadeia de abastecimento, como o transporte, processamento e preparação de alimentos, bem como o desperdício e o lixo gerado ${ }^{6,7}$.

Voltando o olhar para o Brasil, segundo os Indicadores de Desenvolvimento Sustentável do Instituto Brasileiro de Geografia e Estatística (IBGE) ${ }^{8}$, o País está entre os dez maiores emissores de GEE para a atmosfera, além de estar destruindo a vegetação natural e ameaçando a extinção de espécies, com destaque para o desmatamento da Amazônia e as queimadas no cerrado, e contaminando rios por efluentes domésticos e industriais.

A partir desse contexto, é necessário repensar o consumo de alimentos e, principalmente as dietas. O sistema alimentar não tem sido delineado para favorecer a saúde pública e o meio ambiente. No entanto, pode ser influenciado pelo consumo, pois o que e o quanto comemos 
impacta diretamente o que e quanto produzimos. Portanto, é necessário que se reflita sobre o que são dietas adequadas diante do contexto atual e futuro. Ou seja, o que seriam dietas adequadas no século XXI?

Para tentar responder a esta pergunta, o objetivo deste ensaio foi o de realizar análise reflexiva sobre o tema das dietas sustentáveis, salientando a necessidade de debater o que seria uma alimentação adequada dado o impacto ambiental do sistema alimentar atual. Inicia-se com uma digressão sobre a construção dessa temática para, na sequência, traçar paralelos entre dietas saudáveis e sustentáveis. Este ensaio é baseado em uma síntese narrativa de publicações científicas e literatura cinzenta de uma gama interdisciplinar de bases de dados.

\section{O que são dietas sustentáveis?}

Nesta seção, o objetivo é o de identificar o surgimento e a definição do termo 'dietas sustentáveis' na literatura com base nos autores e organizações que vêm tentando compreendê-lo. Por ser um tema novo, seu conceito ainda está em fase de construção e aprofundamento, tanto teórica como empiricamente.

O primeiro trabalho publicado a utilizar o termo dietas sustentáveis foi escrito por Gussow e Clancy em $1986^{9}$ no 'Journal of Nutrition Education', com o título 'Dietary Guidelines for Sustainability'. As autoras arguiam que já não era suficiente para a educação nutricional oferecer informações sobre a relação entre saúde humana e escolhas alimentares, pois os consumidores instruídos precisavam fazer escolhas alimentares que não só melhorassem a sua própria saúde, mas também contribuíssem para a proteção dos recursos naturais. Nesse sentido, sugeriram o termo 'dietas sustentáveis', relacionando-o à 'agricultura sustentável', considerando que sustentabilidade estaria associada com qualquer coisa capaz de se manter dentro de sistemas naturais em um futuro previsível.
Assim, uma agricultura sustentável seria aquela que usa recursos humanos e naturais para produzir alimentos e fibras de maneira conservadora, isto é, de uma maneira que não se desperdicem recursos finitos, como o solo, água e energia fóssil. Em outro estudo publicado em 1989 por Herrin e Gussow ${ }^{\mathbf{1 0}}$, as autoras se propõem a discutir uma dieta regional sustentável, defendendo a ideia do consumo de alimentos produzidos localmente.

Já na década de 1990, Feenstra"1 retoma o argumento de que a construção de um sistema alimentar sustentável e seguro começa reconectando os sistemas alimentares locais com a construção de uma comunidade saudável, chamando a atenção para o termo Nutrição Ambiental. Tal termo estaria relacionado à ideia de que as escolhas alimentares têm consequências e podem fazer diferença na forma em que os recursos naturais são usados. Wahlqvist et al.12 utilizam o termo Econutrição para fazer alusão à importância da variedade alimentar e biodiversidade para a saúde e para o ambiente.

No final da década de 1990, Erdmann et al. ${ }^{13}$, usando o termo consumo alimentar sustentável, defendiam que o mesmo era baseado em quatro dimensões: econômica, ambiental, saúde e social. No entanto, eles não davam nenhuma orientação sobre como essas facetas deveriam ser ponderadas ao colocar em prática o consumo sustentável de alimentos. Tentando tratar de forma pragmática, Leiztmann ${ }^{\mathbf{1 4}}$ afirmava que o consumo sustentável de alimentos deveria ser definido como a preferência por dietas sem carne ou com quantidades reduzidas, juntamente com alimentos orgânicos, regionais e sazonais minimamente processados, embalados ecologicamente, preparados com bom gosto e comercializados com justiça.

Mas retomando o termo dietas sustentáveis, o estudo de Wallén et al. ${ }^{15}$ perguntava: 0 que torna uma dieta sustentável? Os autores citavam um artigo na revista sueca 'New Scientist' de 1997, que definia dietas sustentáveis como aquela que usava eficientemente a energia. Porém, esses autores criticavam tal simplificação, e sua definição de dieta 
nutricionalmente sustentável seria aquela que fornecesse a quantidade necessária de nutrientes e energia para manter uma boa saúde, além de considerar o aspecto ambiental, que poderia ser medido a partir de uma série de critérios, como uso da terra, gestão de resíduos, emissão de GEE, ou diversidade biológica.

A partir desse momento, segundo Weber e Matthews ${ }^{16}$, estudos vinham demonstrando que os produtos de origem animal, principalmente carnes vermelhas, eram os gêneros com maiores impactos ambientais. Portanto, os mesmos autores relatavam que comer menos carne vermelha e produtos lácteos seria uma maneira mais eficaz de reduzir esses impactos do que comprar alimentos locais.

No entanto, um artigo de 2011 escrito por Burlingame e Dernini ${ }^{17}$ defendia que o conceito de dietas sustentáveis teria sido negligenciado por muitos anos, desde sua criação em 1986 por Gussow e Clancy ${ }^{9}$, e que o processo de globalização alimentar e o aumento da produção de sistemas agrícolas desconsiderara a sustentabilidade dos ecossistemas. Portanto, o interesse em dietas sustentáveis estaria sendo retomado por sociedades científicas internacionais e instituições de vários governos europeus. Estavam se referindo ao debate sobre sustentabilidade em 2010 , onde a FAO cunhou o conceito de 'Dietas Sustentáveis':

São aquelas com baixo impacto ambiental que contribuem para a segurança alimentar e nutricional e para uma vida saudável das gerações futuras. As dietas sustentáveis são protetivas e respeitadoras da biodiversidade e dos ecossistemas, culturalmente aceitáveis, economicamente acessíveis e justas, nutricionalmente adequadas, seguras e saudáveis, enquanto otimizam recursos naturais e humanos ${ }^{\mathbf{1 8 ( 7 )}}$

A defesa das dietas sustentáveis ${ }^{\mathbf{1 8 , 1 9}}$ advém da integração dessa noção com a abordagem da cadeia alimentar, ou seja, 'levar a biodiversidade da fazenda para o prato’. Essa integração deveria guiar um esforço intersetorial inovador para contrabalançar a simplificação das dietas, a perda de biodiversidade e a degradação dos ecossistemas e evitar mais erosão das culturas alimentares. Como tal, a definição reconhece o papel da nutrição para uma vida saudável, agora e no futuro, enfatizando a dimensão de tempo na compreensão da segurança alimentar. Também sublinha o papel do consumo sustentável como motor da produção sustentável.

Porém, para Meybeck e Gitz ${ }^{\mathbf{2 0}}$, a definição da FAO não envolve o sistema alimentar diretamente, e, portanto, os autores propõem outra definição:

Uma dieta sustentável é uma dieta que contribui para o bom estado nutricional e a boa saúde no longo prazo do indivíduo/comunidade e isto contribui para e é ativado por sistemas alimentares sustentáveis, contribuindo assim para a segurança alimentar e nutricional no longo prazo ${ }^{20(3)}$.

Os autores defendem que essa definição relaciona explicitamente as duas noções e transforma as dietas sustentáveis em um conceito e também em um objetivo: transformar o sistema alimentar para alcançar segurança alimentar e nutricional.

Para Mason e Lang', o termo se refere a ambos, saúde e ambiente, e contém uma multiplicidade de metas para uma alimentação saudável, de forma a causar o menor impacto ambiental, indo ao encontro da questão cultural e socioeconômica. No entanto, para os autores, essa abordagem é bastante complexa. Concordam com outros autores ${ }^{6}$ que sustentabilidade é um conceito que significa diferentes coisas para diferentes pessoas e que ele pode ser tratado de forma suave ou mais contundente. Assim, ao decidir consumir um alimento por alguma característica mais saudável ou sustentável, está se fazendo uso de um conceito suave de sustentabilidade. Já por outro lado, se a escolha for a de mudar rigorosamente o que se come diariamente sob todos os aspectos do que se considera sustentável, já é um uso mais enfático do que se considera um comportamento sustentável. 
Somada a essa complexidade, está o que se entende por dieta ou alimentação. Comida não é apenas algo que se faz uso para sobreviver, mas também é um ato cultural, onde as escolhas, os gostos, os hábitos delineiam o que se pode comer, o que é preferido, o que é cotidiano, de acordo com a religião, etnia, região etc. Também envolve o aspecto econômico, pois os preços, a renda, o acesso são determinantes dos produtos que compõe a alimentação. As dietas, portanto, ampliam a perspectiva de algo comestível para outra mais abrangente, dado que ela se define pela quantidade total de ingestão de alimentos durante um período de tempo. Portanto, Mason e Lang ${ }^{21}$ chamam a atenção para o fato que ela diz respeito a um padrão, hábito, aculturação que se delineia com o tempo.

Quando se juntam dois termos complexos, dietas e sustentabilidade, obviamente, a complexidade aumenta. Assim sendo, falar de dietas sustentáveis no Brasil é diferente de falar disso em outros países. O que seria uma dieta sustentável aqui, considerando a cultura, a região, a religião, a renda, o ambiente, não será uma dieta sustentável no Reino Unido, pois essas características são variáveis. Dessa forma, as análises, os indicadores, os dados para medir a sustentabilidade das dietas são diferentes nos diferentes locais.

Salientar a complexidade do tema visa a não cair na armadilha do simplismo e do cartesianismo, estudando partes separadas de um sistema que funciona interligado. No entanto, em uma tentativa de esclarecer melhor o que seriam dietas sustentáveis, tentou-se entender melhor suas dimensões, pautando-se nos quatro domínios - saúde, economia, sociedade e meio ambiente - de um sistema alimentar sustentável22. As dietas sustentáveis dialogariam com essas dimensões, quando se advoga que devam ser culturalmente aceitáveis, i.e., dimensão social; acessíveis e justas - dimensão econômica; seguras, nutritivas e saudáveis - dimensão da saúde; e protetivas e respeitadoras da biodiversidade e dos ecossistemas - dimensão ambiental. Além dessas dimensões, a definição da FAO e de outros autores que vêm estudando a temáti$\mathrm{ca}^{23}$ adicionam a agricultura como um quinto determinante ou uma quinta dimensão.

Tais cinco dimensões ou determinantes devem ser entendidos como interconectados e, seguindo a argumentação de alguns autores ${ }^{6,20-}$ ${ }^{24}$, correspondem a um conjunto de questões. A dimensão da saúde está relacionada com o sistema alimentar, pois depende dele para ter disponibilidade de alimentos em quantidade suficiente e de qualidade. Nosso organismo precisa de determinado volume de nutrientes específicos que só podem ser alcançados quando a sociedade tem um sistema que os produz. No momento em que esse sistema passa a produzir menos diversidade de alimentos, com menor qualidade nutricional e com maior densidade energética, a saúde é prejudicada em função das deficiências de determinados nutrientes ou substâncias ou excesso de outros.

Portanto, as altas prevalências de excesso de peso, obesidade, desnutrição, anemia, diabetes, doenças cardiovasculares e outras patologias que países desenvolvidos, mas principalmente pobres, vêm sofrendo revelam que a disponibilidade de alimentos do sistema alimentar atual está em dissonância com a saúde pública. A produção, processamento e distribuição dos gêneros alimentícios tem se tornado um risco às dietas, já que os produtos mais acessíveis em termos de preços são, frequentemente, os menos saudáveis e nutritivos, afetando particularmente a população mais vulnerável. Por outro lado, o sistema alimentar pode afetar e ser afetado por questões ambientais que culminam com prejuízos à saúde, como, por exemplo, a dificuldade de acesso à água potável e a perda da biodiversidade, 6,20-24.

A dimensão econômica está implicitamente vinculada com a saúde, como já referenciado. Nações mais ricas e com melhor distribuição de renda tendem a proporcionar à sua população mais condições de acessar alimentos em quantidades suficientes e com qualidade nutricional superior. No entanto, isso não pode 
ser considerado uma regra, já que problemas de saúde consequentes da dieta estão aumentando nesses locais também. Além do acesso, a dimensão econômica também abrange as políticas econômicas internacionais e nacionais. Um exemplo são os subsídios dados em determinados países para produtos como grãos para aumentar a sua produção e processamento, tornando-os mais baratos e competitivos no mercado global, mas, invariavelmente, menos nutritivos e que são depois vendidos a países mais pobres. Essa política não só prejudica pelo tipo de produto ofertado, mas também pelo prejuízo causado às economias locais dos países receptores ${ }^{6,20-24}$.

A relação das dietas sustentáveis com as questões socioculturais é determinante. A religião, em alguns locais do mundo, é fortemente influenciadora no que diz respeito às prescrições e proscrições alimentares, a exemplo da Índia, onde o hinduísmo delineia uma dieta mais propensa ao vegetarianismo. No outro extremo, países da América Latina, como Uruguai, Argentina são mais aderentes a uma dieta mais rica em carnes, não por questões religiosas, mas por uma geografia propícia à criação de animais. Já no México, Peru, Bolívia, pode-se verificar o hábito ancestral do uso substancial de cereais, como o milho.

No entanto, atualmente, a cultura tem sido fortemente moldada pela mídia e os padrões alimentares têm se tornado cada vez mais homogeneizados e com menor diversidade de produtos. Alimentos básicos e que faziam parte da cozinha de determinados povos foram perdendo espaço para produtos industrializados e altamente processados. Exemplo disso são as comidas típicas brasileiras, como a mandioca, o arroz e o feijão, trocadas por fast food e outros produtos mais práticos e com maior apelo midiático ${ }^{6,20-24}$.

A dimensão ambiental das dietas sustentáveis está associada com o impacto do que comemos no desequilíbrio do ecossistema e vice-versa, como uma via de mão dupla. As escolhas alimentares de uma população influenciam o quanto usamos de recursos finitos, como água, terra e produtos fósseis para produção de energia e insumos químicos, no sistema alimentar e quanto esse processo libera de gases tóxicos, reduz a biodiversidade, produz de lixo e contamina solos e água. Nesse sentido, as práticas de produção e consumo de alimentos estão colocando pressões sem precedentes sobre o ambiente natural e alterando os ecossistemas onde as pessoas vivem, o que acaba afetando profundamente suas dietas. O uso insustentável desse capital natural leva a problemas ambientais que se refletem na instabilidade climática e, por sua vez, na produção de alimentos. Escassez ou excesso de chuvas, por exemplo, afetam as culturas agrícolas, inviabilizando colheitas e aumentando consideravelmente os preços dos alimentos, o que toca no quesito da acessibilidade econômica, já discutida ${ }^{6,20-24}$.

Finalmente, para fechar a interrelação entre os diferentes domínios do que seria considerada uma dieta sustentável, está a agricultura. A decisão do que produzir, quanto e como não tem respeitado princípios de saúde e sustentabilidade, mas tem, essencialmente, objetivado lucros econômicos. Tem-se tratado a agricultura como produtora de comodities agrícolas, como setor econômico responsável pela produção de riqueza dos países e objetiva-se melhorá-la a partir de incrementos de produtividade, substituindo ou se apropriando da natureza. A revolução verde foi um reflexo dessa perspectiva, com o aumento de tecnificação e da industrialização do campo. Nessa trajetória, pouca atenção foi dada a suas consequências na saúde pública e na sustentabilidade do sistema do ponto de vista social e ambiental.

Portanto, produzem-se mais alimentos não saudáveis do que saudáveis, de forma que estes últimos se tornam mais inacessíveis e os primeiros impõem-se como padrão cultural. Além disso, a forma de produzir do sistema agroalimentar está assentada no uso indiscriminado dos recursos naturais sem nenhum pagamento ambiental pelos danos criados. A erosão do solo, a contaminação das águas, a perda da biodiversidade, o uso indiscriminado 
de fertilizantes e pesticidas são alguns dos problemas causados pelo sistema convencional.

Novas formas de produzir são imperativas, indicando a necessidade de modelos agroecológicos de agricultura. Além da produção de alimentos, o sistema alimentar é composto por uma cadeia de processos que envolvem o transporte, processamento, embalagem e preparo. Essas fases, a depender do produto que se está considerando, podem emitir altas quantidades de GEE ${ }^{6,20-24}$.

Portanto, o conceito de dietas sustentáveis sugere que elas devem ser constituídas de forma a ter o potencial de recalibrar o atual sistema alimentar, que compromete a capacidade de produzirem alimentos no futuro e pode trazer efeitos irreversíveis no ecossistema e na saúde. É importante reconhecer que o mito do século XX de que os humanos poderiam comer o que e quanto quisessem sem consequências para ninguém a não ser para si mesmos, deve ser derrubado, pois o planeta tem seus limites ${ }^{6,21}$.

\section{Dietas sustentáveis e saudáveis são convergentes?}

Na literatura internacional, alguns autores, ${ }^{6,21,25}$ têm tentado responder esta pergunta - dietas saudáveis são também dietas sustentáveis ou quais os pontos de concordância e discordância? E qual o alinhamento das dimensões socioculturais e econômicas?

Segundo as diretrizes nutricionais ${ }^{26}$, o grupo de alimentos que deveria compor a maior parte da dieta, seja por seu conteúdo em energia - cereais, tubérculos e derivados - ou por seu conteúdo de vitaminas, minerais e fibras - hortaliças e verduras -, seriam os alimentos vegetais. Recomenda-se que esses produtos sejam consumidos, preferencialmente, de forma integral e não refinada, dado seu conteúdo de fibras e seus benefícios à saúde e prevenção de doenças. Em relação ao ambiente, esses alimentos, i.e., milho, trigo, batatas, têm demonstrado baixo impacto ambiental, pois têm baixa emissão de GEE, com exceção do arroz, que requer irrigação e gera altos níveis de metano ${ }^{25}$. No entanto, algumas culturas recebem altas concentrações de fertilizantes e agrotóxicos e ainda se constituem como grandes monoculturas, a exemplo do milho e do trigo no País27, o que pode prejudicar uma gama de serviços ecossistêmicos ${ }^{28}$.

A dimensão socioeconômica desse grupo de alimentos ainda é pouco investigada, mas, segundo Garnett ${ }^{6}$, merece mais atenção. Nesse sentido, há que se salientar a perda do patrimônio genético das sementes com o advento da transgenia e da monocultura. O mercado de sementes tem se tornado cada vez mais monopolizado, visto que apenas quatro empresas dominam esse mercado no mundo ${ }^{29}$, revelando elevado grau de insustentabilidade econômica e sociocultural de seus produtos.

Em relação às frutas e hortaliças, verifica-se que produtos menos sensíveis, como cenoura, beterraba, maçã etc. produzem menos GEE. Assim, as que precisam mais cuidados pela sua fragilidade, como as que necessitam de proteção para ser produzidas, como tomates, morangos, ou que necessitam refrigeração, como as folhosas, produzem mais GEE25. Por outro lado, essa não é única preocupação, pois algumas variedades precisam de mais água ou são mais suscetíveis a pragas, levando ao maior uso de agrotóxicos. Segundo Kramer et al. ${ }^{30}$, analisando o consumo holandês, o grupo de cereais, tubérculos, frutas, hortaliças contribuíam com $55 \%$ da emissão de óxido nitroso (N2O) das compras de alimentos. Segundo os autores, a produção e aplicação de fertilizantes nitrogenados sintéticos na agricultura são responsáveis por grande parte das emissões de $\mathrm{N} 2 \mathrm{O}$ nos ciclos de vida dessas categorias.

Outra discussão muito realizada na literatura é a produção local e sazonal dos alimentos, considerando que ambos afetariam o impacto ambiental. Segundo Garnett ${ }^{6}$, observações identificaram que produções locais fora de época podem emitir mais gases do que as 
produzidas sazonalmente em outros locais, indicando que o fator sazonalidade é mais importante que o fator localização. Já no que tange ao uso de energia, frutas e verduras produzidas fora do País e transportadas por navio ou avião podem chegar a ter um uso energético em torno de $65 \%$ a mais do que o necessário, no caso da produção de carne de gado ${ }^{25}$.

É também importante frisar que muitos desses produtos acabam sendo desperdiçados, talvez porque sejam altamente perecíveis, mas principalmente porque não estão sendo consumidos. Pesquisa realizada no Brasil ${ }^{\mathbf{3 1}}$ tem identificado, nas últimas décadas, um declínio no consumo de hortaliças e frutas, sinalizando que apenas $10 \%$ da população tem consumido a quantidade indicada pela Organização Mundial da Saúde.

Em relação às questões socioeconômicas, o Brasil é um grande produtor dessas hortifrutis, em boa parte, cultivadas por agricultores familiares, tendo se destacado como um exportador de frutas tropicais como uva, manga, banana, abacaxi, entre outros ${ }^{32}$. Além disso, frisa-se a diversidade desses produtos em todo território nacional ${ }^{32}$, configurando-se alimentos superiores do ponto de vista das dietas sustentáveis.

Já os produtos de origem animal, como carnes, peixes e derivados, ricos em proteínas, ferro, zinco, riboflavina, vitamina B12, vitamina A e vitamina $\mathrm{D}$, também são fontes importantes de ácidos graxos saturados, o que estaria associado a doenças cardiovasculares. Além disso, estudo ${ }^{33}$ indica que alto consumo de carne vermelha e processada em combinação com uma baixa ingestão de fibra alimentar tem sido associada com o aumento do risco de câncer colorretal.

Além disso, esse grupo de alimentos, principalmente as carnes, têm sido responsabilizados como os campeões no que tange aos impactos ao meio ambiente. As carnes são o principal grupo de alimentos investigados pelos pesquisadores, especialmente a vermelha. Em pesquisa na Holanda ${ }^{30}$, o consumo doméstico de carnes e leites respondeu por $85 \%$ da emissão de gás metano na atmosfera e em torno de $45 \%$ de emissão de dióxido de carbono (CO2), confirmando o impacto desse produto no ambiente em conjunto com outros estudos ${ }^{33,34}$.

A pecuária e a agricultura são responsáveis por $70 \%$ do consumo de água doce no planeta, sendo que, a maior parte é usada para irrigar cereais, oleaginosas ou leguminosas, i.e., soja, girassol, algodão, linhaça etc., que são, por sua vez, usadas como alimentos e proteínas na alimentação do gado. Além disso, a água doce é usada para hidratar o gado, limpar estábulos, salas de ordenha e abatedouros ${ }^{35}$. Em relação ao uso da terra, estudo ${ }^{34}$ revela que as carnes, principalmente a de gado, utilizam em torno de 27 a $49 \mathrm{~m}^{2}$ para produzir um quilo do produto.

Ainda, as carnes não são eficientes conversoras de calorias. Como os animais são alimentados por ração, são necessários grandes aportes de grãos para seu desenvolvimento. Portanto, se os animais fossem considerados 'máquinas de produção de alimentos', essas máquinas acabariam sendo extremamente poluentes, por ter um alto consumo e ser muito ineficientes. Quando leguminosas são transformados em proteínas animais, a maioria das proteínas e energia contidas nos vegetais é desperdiçada, já que os vegetais consumidos como ração são utilizados pelos animais para seus processos metabólicos, assim como para construir tecido como ossos, cartilagem, miudezas e fezes ${ }^{35}$.

Segundo Peters et al. ${ }^{36}$, o consumo global de carne e outros produtos animais aumentou ao longo da segunda metade do século XX e provavelmente continuará a aumentar no futuro, projetando-se que o consumo cresça $65 \%$ entre 1993 e 2020, i.e., de 184 para 303 milhões de toneladas métricas.

Em relação aos aspectos socioeconômicos e culturais, no Brasil, essa questão fica ainda mais complicada, já que a cultura alimentar é baseada na carne, e o País se destaca como um dos maiores consumidores, produtores e exportadores mundiais de carne bovina, aves e suínos ${ }^{37}$. Também há que se pontuar a importância que o País tem na produção de grãos, como milho e soja para exportação, 
que tem por finalidade a confecção de ração para animais. Sendo o agronegócio um dos principais motores da economia brasileira, há um grande paradoxo no País no que tange à carne. Por um lado, depende-se dela para gerar riqueza, embora, por outro lado, sua produção e consumo causem grandes danos ao ambiente ${ }^{30}$.

Em relação ao consumo de peixes e frutos do mar, há muitas evidências científicas que indicam os benefícios para a saúde no que diz respeito ao seu conteúdo de proteínas, micronutrientes e, especialmente, ácidos graxos ômega 3 - de melhor biodisponibilidade do que em outras fontes -, relacionados a efeitos protetores que têm demonstrado contra doenças cardíacas e outras patologias ${ }^{6}$. No entanto, estudos $6,35,38$ indicam que os estoques marinhos estão diminuindo e que não há peixes suficientes para alimentar o mundo segundo as orientações dietéticas, o que acabou por colocar as recomendações dietéticas para a ingestão de peixe como o conflito mais amplamente reconhecido entre saúde e sustentabilidade ambiental. Mitchell ${ }^{39}$ advoga que o consumo de frutos do mar deve ser diversificado, já que $80 \%$ dos peixes que os europeus compram são de apenas cinco espécies. Além disso, deve-se melhorar a gestão da cadeia de fornecimento para reduzir o desperdício, que pode responder por até $50 \%$ da oferta desses produtos.

Juntamente com carnes, leguminosas, ovos e derivados estão os laticínios - leites e derivados -, que também respondem como fontes de proteínas e outros micronutrientes, principalmente o cálcio e também a vitamina B12. No entanto, assim como a carne, o leite também produz elevadas quantidades de GEE, causando danos ambientais, como já visto acima. Porém, evidências em países europeus de que o leite é uma fonte barata de vitamina B12 e riboflavina e tem um balanço favorável entre a densidade de nutrientes e o impacto ambiental, tem sugerido seu consumo moderado ${ }^{40}$.

Já os alimentos ricos em gorduras e açúcares e pobres em nutrientes, relacionados com problemas de obesidade e de Doenças
Crônicas Não Transmissíveis (DCNT), têm impacto ambiental considerado baixo, embora, em alguns casos, como a produção de cana de açúcar, haja uma grande necessidade hídrica e deflorestação - como é o caso do Brasil desde o seu descobrimento. Há também o uso de milho para produção de glicose, e nesse caso, como já discutido anteriormente, há o uso indiscriminado de agrotóxicos, além do uso de grandes extensões de terra. Assim também pode-se dizer do cacau e do café. Do ponto de vista nutricional, esses produtos não são indispensáveis, e o seu excesso pode causar doenças, o que leva a concluir que o uso que fazem de recursos naturais, como água, terra e energia, são desperdiçados ${ }^{41}$.

Em estudo dinamarquês ${ }^{\mathbf{4 2}}$, cerveja, vinho e álcool contribuem com $9 \%$; doces, com $7 \%$; e café, chá e cacau, com $6 \%$ do total de GEE da dieta média. No agregado desses produtos, a dieta dinamarquesa contribuiria com $22 \%$ de GEE só com esse tipo de produto, o que representa mais da metade da participação de $37 \%$ da carne. Simulações feitas revelaram que reduzir tal consumo pela metade seria tão eficiente quanto reduzir o consumo de carne em $30 \%$, no que diz respeito às emissões de GEE.

Por outro lado, há as questões culturais e socioeconômicas, pois esses alimentos fazem parte da cozinha brasileira e são altamente prazerosos. Além do que o Brasil é também um grande produtor e exportador desses gêneros. Por seu turno, essa produção é muitas vezes caracterizada como grandes monoculturas e latifúndios que, mormente, fazem uso de mão-de-obra barata para o trabalho rural. Há que se salientar que muitas vezes o produto é exportado em sua forma bruta para outros países, não fomentando a indústria processadora geradora de empregos, de tecnologias e de valor agregado 43

Essas evidências científicas indicam uma congruência entre dietas saudáveis e sustentáveis, pois, em linhas gerais, mostram que alimentos que devem ser os mais consumidos, i.e., vegetais, também são os que menos impactam o ambiente e vice-versa. Porém, 
estudos $\mathbf{4 4 , 4 5}$ descobriram que a mudança para uma dieta mais saudável não resulta automaticamente em menor impacto ambiental. Estudo de Macdiarmid et al. ${ }^{46}$ destacou que as recomendações dietéticas para a saúde se baseiam principalmente nas exigências nutricionais, que podem ser alcançadas por meio de várias combinações de alimentos, enquanto o impacto ambiental está associado à redução do consumo de alimentos específicos, como, por exemplo, carne e laticínios.

Segundo os autores, existem sinergias aqui, já que, por exemplo, uma redução na ingestão de carne e produtos lácteos pode beneficiar a saúde, porque, juntos, esses produtos contribuem com $0,50 \%$ da ingestão total de ácidos graxos saturados na dieta do Reino Unido, e um alto consumo de carne vermelha e processada tem sido associada ao câncer. No entanto, qualquer redução nesses alimentos precisa ser considerada no contexto de toda a dieta para garantir que as substituições feitas sejam apropriadas para a saúde. Se isso não for observado e o enfoque se transformar em apenas reduzir GEE, os produtos de origem animal podem ser substituídos por produtos mais baratos, com alto teor de gordura ou açúcar, que tendem a ter menos GEE do que produtos baseados em animais, prejudicando o aspecto da saúde.

Além disso, Carlsson-Kanyama e González ${ }^{25}$ mostram que uma refeição vegetariana com legumes importados poderia usar mais energia e causar maiores emissões de equivalentes de dióxido de carbono do que uma refeição produzida domesticamente à base de carne. Portanto, a discussão não pode ser generalizada, pois onde, como e quando os alimentos são produzidos, são fatores importantes.

Assim sendo, dietas sustentáveis propõem harmonizar as duas preocupações de saúde e ambiente, conciliando-as com as questões econômicas e culturais na escolha dos alimentos que compõem a alimentação diária. Nesse sentido, iniciativas com novas diretrizes e guias nutricionais vêm sendo criadas ao redor do mundo, como a dupla pirâmide do Instituto Barilla ${ }^{47}$, o guia alimentar brasileiro ${ }^{26}$ e as recomendações nutricionais do Qatar ${ }^{48}$, Suécia e Alemanha ${ }^{49}$, reforçando a necessidade de pensarmos nas dietas de forma mais complexa que apenas o aspecto nutricional e de saúde.

\section{Considerações finais}

Em nível teórico, a definição de dietas sustentáveis vem sendo construída conjugando fatores que já existiam, enquanto preocupações, como os aspectos nutricionais, socioculturais, econômicos, a outros que se ligam ao sistema agroalimentar e ao meio ambiente. No século XXI, o que comemos tem grandes impactos ambientais para que continuemos alheios a essas consequências. Nesse sentido, esses resultados estão ligados ao alcance presente e futuro da Segurança Alimentar e Nutricional (SAN), já que se continuarmos no ritmo atual, há grandes riscos de sofrermos com a falta de recursos naturais e de mudanças climáticas que afetarão a produção de alimentos.

O momento para garantir um suprimento sustentável de alimentos para as gerações futuras deve ser pensado seriamente, já que, sem mudanças, o sistema alimentar global continuará a degradar o meio ambiente e comprometerá a capacidade mundial de produzir alimentos no futuro, além de contribuir para a mudança climática e a destruição da biodiversidade. Um grande desafio para ajudar a resolver esse problema é equilibrar a demanda futura e a oferta de produtos agrícolas, o que inclui a necessidade de mudar as dietas das pessoas para escolhas alimentares mais sustentáveis. Assim, aos desafios da fome e das deficiências nutricionais por um lado, e ao do sobrepeso e DCNT por outro, soma-se o desafio de pensarmos na alimentação relacionando-a à sustentabilidade para que possamos no futuro termos garantia de SAN.

No nível pragmático, é provável que não exista uma dieta sustentável, mas sim uma série de padrões alimentares mais sustentáveis do que outros. Tomando por exemplo a 
proteína, existem valores de referência dietéticos para ingestão que podem vir de proteínas animais ou vegetais, mas a escolha feita irá variar significativamente em termos de impactos ambientais.

Nesse sentido, não se pode presumir que uma dieta saudável que atenda aos requisitos de nutrientes terá necessariamente menos emissões de gases, uso de terra, água e energia, uma vez que dependerá das escolhas de alimentos específicos da dieta. Mesmo dentro desses padrões alimentares, haverá alguns alimentos mais sustentáveis do que outros, baseados em fatores da cadeia de suprimentos, dependendo de como e onde os alimentos foram produzidos, fabricados, transportados, preparados e consumidos. Essa variação também é evidente dentro do mesmo grupo de alimentos, ou seja, não é o produto em si o problema, mas o processo pelo qual ele passou do campo ao prato, como método de cultivo intensivo ou extensivo, região geográfica Brasil ou outros países -, método de transporte por fretes aéreos ou embarcados e condições de crescimento i.e, ao ar livre ou em estufas.

Em linhas gerais, os autores estudados concordam que uma dieta com baixo impacto ambiental é geralmente consistente com uma boa nutrição. As dietas mais sustentáveis conteriam elevada proporção de plantas e baixa proporção de animais e seriam extremamente heterogêneas. Tudo isso é precisamente o que os nutricionistas modernos defendem: alto teor de fibras e micronutrientes; a maior parte da energia de carboidratos; proteína modesta; baixa gordura saturada; uma variedade de gorduras insaturadas. Do ponto de vista cultural, as grandes cozinhas do mundo são ricas em cereais - cereais, leguminosas, tubérculos -, fazem o uso do máximo de frutas e legumes disponíveis, são poupadores no uso de carne, usada como guarnição, principalmente em festas; e são tão diversas quanto possam ser concebidas.
A compreensão sobre o impacto ambiental das escolhas alimentares está aumentando, mas o maior desafio continua sendo mudar a atual dieta não saudável e insustentável. Reconhece-se que mudanças substanciais também precisarão ser feitas nas práticas agrícolas e na indústria de alimentos para mudar o ambiente alimentar, mas isso não deve ser visto como uma alternativa para os indivíduos não mudarem seus padrões alimentares, pois é necessária ação em todos os setores.

Por fim, ainda não é conhecido e, consequentemente, não se está atualmente em condições de dar orientações claras aos consumidores sobre essa questão. É uma problemática complexa e multifatorial e requer uma abordagem baseada em evidências para resolver. Além disso, é importante que os nutricionistas da saúde pública continuem a expressar a necessidade de que fatores de sustentabilidade e de saúde sejam considerados em conjunto, a fim de garantir um suprimento de alimentos sustentável e saudável para as gerações futuras.

Embora os nutricionistas estudem a conexão entre orientação alimentar e saúde humana há décadas, estão apenas começando a explorar a conexão entre orientação alimentar e proteção ambiental. De fato, diretrizes e políticas alimentares baseadas em alimentos devem dar a devida consideração à sustentabilidade ao estabelecer metas voltadas para a nutrição saudável. Já foi dado o primeiro passo com o guia alimentar brasileiro, mas precisamos avançar tanto em pesquisa como em políticas e ações que fomentem mais conhecimentos e mais práticas em prol da saúde e da sustentabilidade.

\section{Colaboradora}

Triches RM (0000-0002-4460-4821)* é responsável pela elaboração do manuscrito. 


\section{Referências}

1. Triches RM, Hawkes C. Sustainable diets: Definition, state of the art and perspectives for a new research agenda in Brazil. Ciênc. Saúde Colet. [internet]. No prelo. [acesso em 2019 dez 19]. Disponível em: http://www.cienciaesaudecoletiva.com.br/artigos/sustainable-diets-definition-state-of-the-art-and-perspectives-for-a-new-research-agenda-in-brazil/17325?id=17325.

2. Alsaffar AA. Sustainable diets: The interaction between food industry, nutrition, health and the environment. Food Scienc. Techn. Inter. 2016; 22(2):102111.

3. Food and Agriculture Organization. Global food losses and food waste - Extent, causes and prevention. Rome: Food and Agriculture Organization; 2011.

4. Ridoutt BG, Hendrie GA, Noakes M. Dietary strategies to reduce environmental impact: a critical review of the evidence base. Adv Nutr. 2017; (8):933-46.

5. Rockström JW, Steffen W, Noone K, et al. Planetary boundaries: exploring the safe operating space for humanity. Eco. Soc. 2009; (14):2-32.

6. Garnett T. What is a sustainable healthy diet? A discussion paper. Reino Unido: Food Climate Research Network; 2014.

7. Godfray C. O desafio de alimentar nove mil milhões de pessoas em 2050. In: Santos JL, Carmo I, Graça P, et al. O futuro da alimentação: ambiente, saúde e economia. Lisboa: Fundação Calouste Gulbenkian; 2013. p.18-32.

8. Instituto Brasileiro de Geografia e Estatística. Indicadores de Desenvolvimento Sustentável. Rio de Janeiro: IBGE; 2012.

9. Gussow J. Clancy K. Dietary guidelines for sustainability. J. Nutri. Edu. 1986; (18):1-5.

10. Herrin M, Gussow JD. Designing a sustainable re- gional diet. J. Nutri. Edu. 1989; (21):270-275.

11. Feenstra G. Local food systems and sustainable communities. J. Alternat. Agricu. 1997; (12):28-26.

12. Wahlqvist ML, Specht RL. Food variety and biodiversity: Econutrition. Asia Pac J. Clinic. Nutri. 1998; 7(3/4):314-9.

13. Erdmann L, Sohr S, Behrendt S, et al. Sustentabilidade e Nutrição (Relatório do Workshop). Berlim Instituto de Estudos do Futuro e Avaliação de Tecnologia $\mathrm{GmbH} ; 2003$.

14. Leitzmann C. Nutrition ecology: the contribution of vegetarian diets. Am. J. Clinic. Nutrit. 2003; 78(3):657S-659S

15. Wallén A, Brandt N, Wennersten R. Does the Swedish consumer's choice of food influence greenhouse gas emissions? Environ. Sci. Policy. 2004; 7(6):525-535.

16. Weber CL, Matthews HS. Food-miles and the relative climate impacts of food choices in the united states. Environ. Sci. Technol. 2008; 42(10):3508-3513.

17. Burlingame B, Dernini S. Sustainable diets: the Mediterranean diet as an example. Public Health Nutr. 2011; 14(12A):2285-2287.

18. Food and Agriculture Organization. International Scientific Symposium: Biodiversity and Sustainable Diets - United Against Hunger. Rome: FAO; 2010.

19. Berry EM, Dernini S, Burlingame B, et al. Food security and sustainability: can one exist without the other? Public Health Nutr. 2015; 18(13):2293-2302.

20. Meybeck A, Gitz V. Sustainable diets within sustainable food systems. Proc. Nutr. Soc. 2017; (76):1-11.

21. Mason P, Lang T. Sustainable diets: how ecological nutrition can transform comsumption and the food system. Reino Unido: Routledge; 2017. 
22. Drewnowski A. The Ecosystem Inception Team. The Chicago Consensus on Sustainable Food Systems Science. Front. Nutr. 2018;(4):1-6.

23. Johnston JL, Fanzo JC, Cogill B. Understanding Sustainable Diets: a descriptive analysis of the determinants and processes that influence diets and their impact on health, food security, and environmental sustainability. Adv. Nutr. 2014; (5):418-429.

24. Auestad N, Fulgoni VL. What current literature tells us about sustainable diets: emerging research linking dietary patterns, environmental sustainability, and economics. Adv. Nutr. 2015; (6):19-36.

25. Carlsson-Kanyama A, González AD. Potential contributions of food consumption patterns to climate change. Am. J. Clin. Nutr. 2009; 89(5):1704S-1709S.

26. Brasil. Ministério da Saúde. Secretaria de Atenção à Saúde. Guia alimentar para a população brasileira. Brasília, DF: Ministério da Saúde; 2014.

27. Bombardi LM. Geografia do Uso de Agrotóxicos no Brasil e Conexões com a União Europeia. São Paulo: FFLCH; USP; 2017.

28. Pires CSS, Pereira FM, Lopes MTR, et al. Enfraquecimento e perda de colônias de abelhas no Brasil: há casos de CCD?. Pesq. agropec. bras. 2016; 51(5):422442.

29. Heinrich Böll Foundation. Agrifood Atlas: Facts and figures about the corporations that control what we eat 2017. Berlin: Heinrich Böll Foundation; Friends of the Earth Europe; Belgica: Brussels; 2017.

30. Kramer KJ, Moll HC, Nonhebel S, et al. Greenhouse gas emissions related to Dutch food consumption. Energy Policy. 1999; (27):203-216.

31. Instituto Brasileiro de Geografia e Estatística. Pesquisa de orçamentos familiares 2008-2009: análise do consumo alimentar pessoal no Brasil. Rio de Janeiro: Instituto Brasileiro de Geografia e Estatística; 2011.
32. Empresa Brasileira de Pesquisa Agropecuária. Pecuária e Abastecimento [internet]. [Brasilia, DF]. [acesso em 2020 jun11]. Disponível em: https://www.embrapa.br/grandes-contribuicoes-para-a-agricultura-brasileira/frutas-e-hortalicas.

33. Wyness L, Weichselbaum E, O'Connor A, et al. Red meat in the diet: an update. Nutr. Bull. 2011; (36):3477.

34. Vries M, Boer IJM. Comparing environmental impacts for livestock products: A review of life cycle assessments. Livest. Sci. 2010; (128):1-11.

35. Baroni L, Cenci L, Tettamanti M, et al. Evaluating the environmental impact of various dietary patterns combined with different food production systems. Eur. J. Clin. Nutr. 2007; (61):279-286.

36. Peters CJ, Wilkins JL, Fick GW. Testing a complete-diet model for estimating the land resource requirements of food consumption and agricultural carrying capacity: The New York State example. Renew. Agric. Food Syst. 2007; 22(2):145-153.

37. Carvalho AM, Galvão Cesar CL, Fisberg RM, et al. Excessive meat consumption in Brazil: diet quality and environmental impacts. Public Health Nutr. 2012; 16(10):1893-1899.

38. Farmery AK, Gardner C, Jennings S, et al. Assessing the inclusion of seafood in the sustainable diet literature. Fish Fish. 2017; (18):607-618.

39. Mitchell M. Increasing fish consumption for better health - are we being advised to eat more of an inherently unsustainable protein? Nutr. Bull. 2011; (36):438-442.

40. Werner LB, Flysjö A, Tholstrup T. Greenhouse gas emissions of realistic dietary choices in Denmark: the carbon footprint and nutritional value of dairy products. Food Nutr. Res. 2014; 58:(20687)n.p.

41. Hadjikakou M. Trimming the excess: environmental impacts of discretionary food consumption in Australia. Ecol. Econ., 2017; (131):119-128. 
42. Saxe H, Larsen TM, Mogensen L. The global warming potential of two healthy Nordic diets compared with the average Danish diet. Clim. Change. 2012; 116(2):249-262.

43. Pereira CS. A produção agrícola por habitantes urbanos no Brasil: Análise dos períodos colonial, imperial e a transição para o século XX. Rev. NERA. 2020; 23(53):263-287.

44. Aleksandrowicz L, Green R, Joy EJM, et al, The Impacts of Dietary Change on Greenhouse Gas Emissions, Land Use, Water Use, and Health: A Systematic Review. PLoS ONE. 2016; 11(11):1-16.

45. Perignon M, Vieux F, Soler LG, et al. Improving diet sustainability through evolution of food choices: review of epidemiological studies on the environmental impact of diets. Nutr Rev. 2016; 75(1):2-17.

46. Macdiarmid JI, Kyle J, Horgan GW, et al. Sustainable diets for the future: can we contribute to redu- cing greenhouse gas emissions by eating a healthy diet? Am. J. Clin. Nutr. 2012; (96):632-9.

47. Barilla Center for Food \& Nutrition. Double Pyramid 2015: Recommendations for a sustainable diet. Parma: Barilla Center for Food \& Nutrition; 2015.

48. Seed B. Sustainability in the Qatar national dietary guidelines, among the first to incorporate sustainability principles. Public Health Nutrition. 2015; 18(13): 2303-2310.

49. Lang T, Mason P. Sustainable diet policy development: Implications of multi-criteria and other approaches, 2008-2017. Proc. Nutr. Soc. 2018; 77(3):331-346.

Recebido em 17/05/2019

Aprovado em 18/06/2020

Conflito de interesses: inexistente

Suporte financeiro: não houve 